\title{
Information at Fingertips
}

\author{
Shyamkumar N. Keshava ${ }^{1}$ Hriday Shah ${ }^{2}$
}

1Department of Radiology, Christian Medical College, Vellore,
Tamil Nadu, India
2Division of Interventional Radiology, Department of Radiology,
University of Texas Southwestern Medical Center, Dallas, Texas,
United States

J Clin Interv Radiol ISVIR 2018;2:69-70

We have witnessed an explosive growth in the use of social media technology during the past two decades. Specifically, WhatsApp has revolutionized the way people communicate with each other. There are several factors that have led to the widespread and worldwide adoption of WhatsApp as a social communication tool. It is highly accessible, easy to use, and has a low cost. Moreover, its use has been enhanced by the evolution of smartphones, storage capacity, and wireless Internet network connectivity. Its reliance on the Internet rather than the traditional phone lines allows for easy and less expensive communication across the borders. It fits well with the motto of techno-savvy millennial generation, "play and learn." The use of WhatsApp within the medical community has paralleled its explosive growth within the community at large.

Specifically, WhatsApp has revolutionized communication within the interventional radiology community. The application is generally used both between individual users and in groups. Within a department, it may be used for sharing case schedule, duty roster, images, videos, literature, knowledge, or medical opinions. Formation of groups, which include experts around the world, allows anyone to quickly obtain a second opinion from an expert in the field. For example, if a patient experiences the unusual complication of a rapidly decreasing hematocrit following angioplasty for renal artery stenosis, the primary interventionalist can quickly consult an expert on a WhatsApp group, who may alert to the possibility of capsular perforation by the guidewire and suggest immediate embolization. This quick and easy knowledge sharing among the interventional radiologists has the potential to transform medical practice around the world. Additionally, trainees within the field can quiz each other to make learning fun while also sharing their experiences.

The use of WhatsApp within the medical community has been extensively studied. A PubMed search on "WhatsApp" in June 2018 yielded more than 540 publications since 2015. These studies span across a multitude of specialties, indicative



of its widespread use. A publication from Mexico mentions how effectively they used a WhatsApp group for stroke management in their hospital at various stages, including patient arrival, alerting the group for imaging, intervention, and rehabilitation. ${ }^{1}$ An orthopaedic team from Ireland compared it with the traditional pager system and concluded that WhatsApp was reliable and improved the efficiency. ${ }^{2}$ Others have reported that WhatsApp was more efficient than the hospital-provided duty pager system. Physicians have used WhatsApp as a communication tool between the health
Address for correspondence Shyamkumar N. Keshava, MBBS, DMRD, DNB, FRCR, FRANZCR, Department of Radiology, Christian Medical College, Vellore, Tamil Nadu, India (e-mail: aparna_ shyam@cmcvellore.ac.in).
DOI https://doi.org/

10.1055/s-0038-1669212.

ISSN 2457-0214.
Copyright $₫ 2018$ by Indian Society of Vascular and Interventional Radiology
License terms

(a) (1) $\ominus \circledast$ 
workers and patients. A publication from Korea mentioned how WhatsApp-based discussions were useful in improving residents' knowledge of postoperative pain management. ${ }^{3}$ Another publication highlights how the appropriate use of mobile devices in radiology has contributed to patient care. ${ }^{4}$ There are several publications reporting the beneficial role of WhatsApp for undergraduate teaching as well., 3

Limiting the number of group members and sharing only anonymized data are required to maintain patient confidentiality. WhatsApp does not store the messages on its servers once the messages are delivered. In addition, the messages are end-to-end encrypted, meaning that WhatsApp and third parties cannot read them. It appears that some institutions have developed basic guidelines regarding the usage of WhatsApp for medical purposes. ${ }^{6}$ Needless to say, protecting patient privacy and institutional data are basic ethical and legal requirements of any physician. For example, if one wishes to share an angiographic image with someone who is not on the care team, any information that can be used to identify the specific patient (name, date of birth, medical record number, etc.) should be removed.

The full potential and limitations of WhatsApp have yet to be identified. For some people, it offers a more friendly and informal environment compared with a highly rigid and professional nature of medical practice. Some worry that it might be too addictive and disruptive to daily work. Are we going to learn more side effects over a period of time? Will there be another app that is specifically geared toward the needs of the medical community?

Overall, WhatsApp has been a technological marvel. While it was developed initially as a social media tool, it plays a major role in patient care. The "value for money" is beyond expectations. It has potential to greatly improve patient care by allowing providers to easily communicate with colleagues around the world and benefit from collective knowledge. While acknowledging and accepting a smart app for professional use, one should always keep in mind the potential limitations and the need to maintain patient confidentiality.

\section{Acknowledgment}

We would like to thank Dr. Balasubramanian Karthikumar, Department of Radiology, Christian Medical College, Vellore, India for the line diagram.

\section{References}

1 Calleja-Castillo JM, Gonzalez-Calderon G. WhatsApp in stroke systems: current use and regulatory concerns. Front Neurol 2018;9:388

2 Ellanti P, Moriarty A, Coughlan F, McCarthy T. The use of WhatsApp smartphone messaging improves communication efficiency within an orthopaedic surgery team. Cureus 2017;9(2):e1040

3 Bakshi SG, Bhawalkar P. Role of WhatsApp-based discussions in improving residents' knowledge of post-operative pain management: a pilot study. Korean J Anesthesiol 2017;70(5): 542-549

4 European Society of Radiology (ESR). ESR paper on the proper use of mobile devices in radiology. Insights Imaging 2018;9(2):247-251

5 Raiman L, Antbring R, Mahmood A. WhatsApp messenger as a tool to supplement medical education for medical students on clinical attachment. BMC Med Educ 2017;17(1):7

6 Ranschaert ER, Van Ooijen PMA, McGinty GB, Parizel PM. Radiologists' usage of social media: results of the RANSOM survey. J Digit Imaging 2016;29(4):443-449 